nuclear distance $r_{e}$. With $\mathrm{CO}, \mathrm{N}_{2}, \mathrm{SiO}$ and $\mathrm{P}_{2}$, the band systems under discussion have been observed in absorption as well as in emission, and the lower $\left({ }^{1} \Sigma\right)$ states are therefore stable ground states. The same is expected, though not yet observed, to be true of CS and PN; that is to say, each of these should be sociation. It is thus somewhat less than that of $\mathrm{N}_{2}$ (recently given as about 7.9 volts $^{4}$ rather than the hitherto accepted value of about 9.0 volts $^{2}$ ) and greater than that of $\mathrm{P}_{2}\left(5 \cdot 0\right.$ volts $\left.^{3}\right)$. Similarly the heats of dissociation of $\mathrm{CS}$ and $\mathrm{SiO}$ (each roughly 8 volts) are less than that of $\mathrm{CO}$ (about 10 volts).

The molecules discussed here are all composed of nitrogen, phosphorus and their immediate neighbours in the periodic table. From the atoms preceding and following these we have other 22-electron molecules about which, however, nothing can yet be stated, namely $\mathrm{BCl}$ (bands observed in the same spectral region but not systematised $^{5}$ ) and AlF (expected band system not yet recorded).

\section{W. JEvons.}

capable of existence as gaseous substances in the absence of an electric discharge; J. Curry and L. and G. Herzberg are further investigating the PN system from this point of view.

The heat of dissociation of the ${ }^{1} \Sigma$ state of $P N$ is estimated as $7 \cdot 8$ volts from a Birge-Sponer linear extrapolation of vibrational energies, and as $6 \cdot 3$ volts from a consideration of probable products of dis-
${ }_{1} J$. Chem. Phys, 1, 749; 1933 (preliminary report), Z. Phys., 86,$348 ; 1933$.

${ }^{2}$ Particulars of these band-systems and of the notation used in the PN paper and in the present article are given in the writer"s "Report on Band Spectra of Diatomic Molecules" (Phys. Soc., 1932) The more recent analysis of SiO bands is by Saper (Phys. Rev., 42, 498 ; 1932) and that of CS bands is by Crawford and Shurcliff (Phys. ev., 43, 766; 1933).

${ }^{3}$ G. Herzberg, Ann. Phys., 15, 677 ; 1932.

- Lozier, Phys. Rev., 44, 575; 1933.

- Lochte-Holtgreven and van der Vleugel, Z. Phys., 70, 188; 1931.

\title{
Biology of Heavy Water
}

$\mathrm{I}^{\mathrm{N}}$ Science of February 16, 1934, Prof. Gilbert N. Lewis summarises the results of certain sporadic attempts to observe the effect of water containing heavy hydrogen, $\mathrm{H}^{2}$, upon living organisms. Experiments have necessarily been confined to small organisms, though some preliminary observations on mice are included. The first experiments were upon tobacco seeds, the germination of which was completely retarded by pure $\mathrm{H}_{2}^{2} \mathrm{O}$ and slowed up some 50 per cent by water containing 50 per cent $\mathrm{H}_{2}^{2} \mathrm{O}$. Seeds transferred to normal water after three weeks in pure $\mathrm{H}_{2}^{2} \mathrm{O}$ sprouted in about half the cases but gave unhealthy seedlings. Yeast cultures in an appropriate nutrient medium dissolved in pure heavy water failed to grow, and Pacsu has also shown that the evolution of carbon dioxide by yeast from sugar solution made up with heavy water is much diminished.

In an experiment that was expensive if preparatory in nature, a mouse was supplied in three doses with some $0.66 \mathrm{gm}$. of pure $\mathrm{H}_{2}^{2} \mathrm{O}$. The mouse survived, though during the experiment it showed "marked signs of intoxication". The symptoms of distress seemed more marked after each dose but not cumulative, which led Prof. Lewis to conclude that the heavy water was being voided, but no preparation had been made to test this point. Prof. Lewis concludes that $\mathrm{H}^{2}$ is not toxic in any high degree but that its complete substitution for $\mathrm{H}^{1}$ leads probably to a complete inhibition of growth, an effect which is to be traced to "the greatly reduced rate of all physicochemical processes when $\mathbf{H}^{2}$ is substituted for $\mathbf{H}^{\mathbf{1}}$."

Mr. S. L. Meyer of the Vanderbilt University Biology Department describes in Science of March 2 culture experiments with a blue mould, in which those grown on media made up with one out of every 214 hydrogen atoms $\mathrm{H}^{2}$ gave sixteen times the yield of fungus as those grown on control solutions free from $\mathrm{H}^{2}$.

The late Dr. Edward W. Washburn and Dr. Edgar R. Smith have been carrying on experiments at the Bureau of Standards at Washington in which they have studied the proportion of $\mathrm{H}^{2}$ atoms present in the tissues after plants have grown in normal soil solutions. So far as could be judged, rooted willow cuttings absorbed $\mathrm{H}^{1}$ and $\mathrm{H}^{2}$ in the proportions in which they were present in the original water supply, but apparently the heavy hydrogen was selectively accumulated in the tissues as the expressed sap contained water $2 \cdot 8$ parts per million heavier than normal water, whilst the water obtained from the destructive distillation of the willows was 5.4 parts per million heavier than the normal supply. Dr. Washburn died suddenly on February 6 ; his report with Dr. Smith has been published since, in Science of February 23.

\section{Universe and Atom}

$\mathrm{T}$ HE issue of Die Naturwissenschaften of March 9 contains the address on this subject which Prof. Wehl of Göttingen gave at the opening of the holiday course on mathematical sciences given at Göttingen in July 1933. His object was to put before his audience only such conclusions as are at the present time reasonably certain and to avoid any fantastic speculations.
By representing space in the space time continuum as the abscissa and time as the ordinate of a point on a curved surface, Prof. Wehl shows how the Einstein continuum with its mass distribution is represented by a cylindrical surface with its axis vertical and its radius determined by the density of distribution of mass. Stars at rest are represented by generating lines and the movement of light 